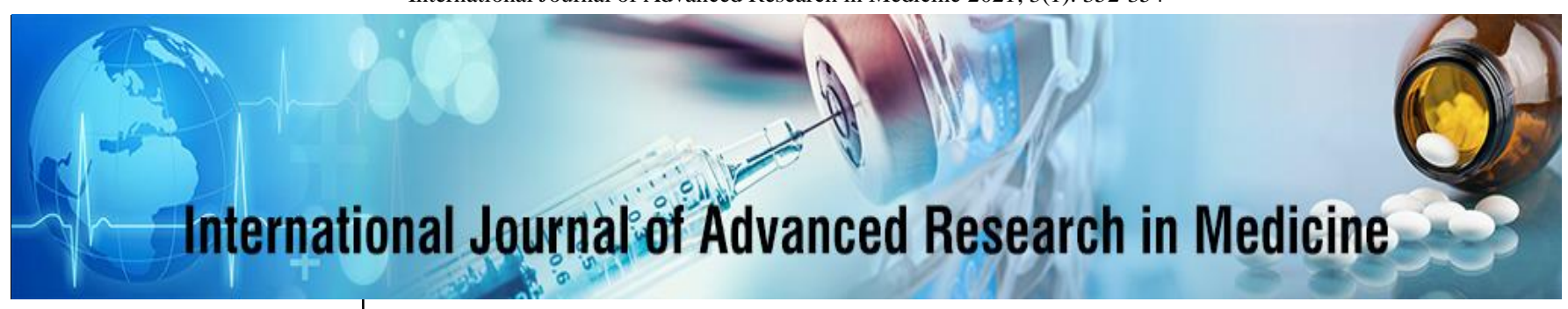

E-ISSN: 2706-9575

P-ISSN: 2706-9567

IJARM 2021; 3(1): 352-354

Received: 10-11-2020

Accepted: 19-12-2020

Dr. Prathibha Vasu

Assistant Professor,

Department of General

Medicine, Dr. B R Ambedkar Medical College, Bangalore,

Karnataka, India

Dr. Parashuram

Associate Professor,

Department of General

Medicine, Dr. B R Ambedkar

Medical College, Bangalore,

Karnataka, India
Corresponding Author:

Dr. Parashuram

Associate Professor,

Department of General

Medicine, Dr. B R Ambedkar

Medical College, Bangalore,

Karnataka, India

\title{
Effects of thyroid dysfunction on lipid profilein postmenopausal women
}

\section{Dr. Prathibha Vasu and Dr. Parashuram}

DOI: $\underline{\text { https://doi.org/10.22271/27069567.2021.v3.i1f.189 }}$

\begin{abstract}
According to the American Association of Clinical Endocrinologists (AACE), millions of women with unresolved menopausal like symptoms, even those taking estrogen may be suffering from undiagnosed thyroid disease. Data was collected using a pretested proforma meeting the objectives of the study. Detailed history and necessary investigations were undertaken. The purpose of the study was explained to the patient and informed consent obtained. Minimum of 100 patients are selected randomly who fulfil the inclusion and exclusion criteria. $36 \%$ of the postmenopausal women had dyslipidemia. Remaining 64\% did not have dyslipidemia. In our study, among 64 patients who did not have dyslipidemia, 8 patients had SH, 5 patients had hypothyroidism, 2 patients had thyrotoxicosis, rest were euthyroid. Among 36 patients with dyslipidemia, 14 patients had SH, 3 patients had hypothyroidism and rest 19 were euthyroid.
\end{abstract}

Keywords: Thyroid dysfunction, lipid profilein, postmenopausal women

\section{Introduction}

Menopause is the permanent cessation of menses, as a result of the irreversible loss of a number of ovarian functions, including ovulation and estrogen production. It is diagnosed retrospectively after twelve consecutive months of amenorrhoea. But transition is not sudden, it takes few years and this transition period is called climacteric or Perimenopause period. The median age for the onset of the climacteric transition is 47.5 years. The median age of menopause is 51 years ${ }^{[1]}$.

Menopause results from loss of ovarian sensitivity to gonadotropin stimulation which is directly related to follicular decline and dysfunction. The oocytes in the ovaries undergo atresia throughout a woman's life and both the quantity and quality of follicles undergo a critical decline approximately 20-25 years after menarche ${ }^{[2]}$. Although fertility declines, pregnancy can still occur as demonstrated by a relatively high rate of unintended pregnancies in women 40-44 years age.

According to the American Association of Clinical Endocrinologists (AACE), millions of women with unresolved menopausal like symptoms, even those taking estrogen may be suffering from undiagnosed thyroid disease. While symptoms such as fatigue, depression, mood swings and sleep disturbance are frequently associated with menopause they may also be signs of hypothyroidism. A survey done by the AACE showed that only one in four women who have discussed menopause \& its symptoms with a physician were also tested for thyroid disease ${ }^{[3]}$.

The thyroid and menopause connection is complex. It actually stems from close interactions between thyroid hormones and reproductive organs because thyroid hormones regulate metabolism, they directly influence the activity of reproductive glands. In addition estrogen and progesterone directly affect thyroid uptake receptor sites by blocking or allowing them to function. Not only do the symptoms of an imbalance of thyroid hormones mirror many of those associated with fluctuating levels of estrogen and progesterone, but the two conditions may be involved in the causal relationship ${ }^{[4]}$. Synthetic hormone use during HRT (Hormone replacement therapy) interact with and affect the functioning of the thyroid as well.

Thyroid dysfunction results in changes in the composition and transport of lipoprotein. Overt and subclinical hypothyroidism is associated with hypercholesterolemia mainly due to elevation of LDL cholesterol, whereas HDL cholesterol is normal or elevated. Hyperthyroidism (both overt and subclinical) is accompanied by decrease in serum levels of 
total LDL and HDL cholesterol. These changes in lipid profile are explained by the regulatory effects of thyroid hormones on the activity of some key enzymes of lipoprotein metabolism ${ }^{[5,6]}$.

\section{Methodology}

Source of Data

Postmenopausal women who fulfil the inclusion and exclusion criteria as mentioned below, attending the outpatient and inpatient of Medicine.

\section{Type of Study}

Hospital based Cross sectional study

\section{Period of study}

One year study period

\section{Method of Collection of Data}

Data was collected using a pretested proforma meeting the objectives of the study. Detailed history and necessary investigations were undertaken. The purpose of the study was explained to the patient and informed consent obtained. Minimum of 100 patients are selected randomly who fulfil the inclusion and exclusion criteria. Relevant history including symptoms and signs at presentation, past medical history, menstrual history, drug history and examination findings was be noted.

\section{Investigations}

Routine Investigations

- Complete blood count

- Blood urea

- Serum creatinine

- Urine analysis

- FBS, PPBS

- ECG

- Chest X ray PA view

- USG abdomen pelvis

\section{Special investigations}

- Thyroid profile - TSH, Total T4.

- Lipid profile - Total cholesterol, Triglycerides, LDL cholesterol, HDL cholesterol.

\section{Results}

Table 1: Prevalence of dyslipidemia

\begin{tabular}{|c|c|c|}
\hline Dyslipidemia & Number $(\mathbf{n}=\mathbf{1 0 0})$ & Percentage \\
\hline Present & 36 & $36 \%$ \\
\hline Absent & 64 & $64 \%$ \\
\hline
\end{tabular}

$36 \%$ of the postmenopausal women had dyslipidemia. Remaining $64 \%$ did not have dyslipidemia.

Table 2: Correlation of Thyroid dysfunction of patients and Dyslipidemia

\begin{tabular}{|c|c|c|c|c|c|}
\hline \multirow{2}{*}{ Dyslipidemia } & \multirow{2}{*}{$\begin{array}{c}\text { Total no. } \\
\text { Of patients }\end{array}$} & Normal & SH & Hypo & Thyrotoxi-cosis \\
\cline { 3 - 5 } & 64 & $49(76.6 \%)$ & $8(12.5 \%)$ & $5(7.8 \%)$ & $2(3.1 \%)$ \\
\hline Absent & 36 & $19(52.8 \%)$ & $14(38.9 \%)$ & $3(8.3 \%)$ & $0(0 \%)$ \\
\hline Present & 100 & $68(68 \%)$ & $22(22 \%)$ & $8(8 \%)$ & $2(2 \%)$ \\
\hline Total & \multicolumn{5}{|c|}{$\begin{array}{c}\text { Dyslipidemia is significantly associated with thyroid } \\
\text { dysfunction with P=0.04*(2x4 fisher exact test) }\end{array}$} \\
\hline \multirow{2}{*}{ Inference } & \multicolumn{5}{|c|}{} \\
\end{tabular}

In our study, among 64 patients who did not have dyslipidemia, 8 patients had $\mathrm{SH}, 5$ patients had hypothyroidism, 2 patients had thyrotoxicosis, rest were euthyroid. Among 36 patients with dyslipidemia, 14 patients had SH, 3 patients had hypothyroidism and rest 19 were euthyroid.

Dyslipidemia is significantly associated with thyroid dysfunction with $\mathrm{P}=0.04 *(2 \times 4$ fisher exact test $)$

Table 3: Correlation of thyroid dysfunction of patients and Pattern of Dyslipidemia

\begin{tabular}{|c|c|c|c|c|c|}
\hline Pattern of Dyslipidemia & Total number of patients & Euthyroid & SH & Hypo & Thyrotoxic \\
\hline Hypercholesterolemia & 36 & $19(52.77 \%)$ & $14(38.88 \%)$ & $3(8.33 \%)$ & 0 \\
\hline Hypertriglyceridemia with hypercholesterolemia & 5 & 0 & $4(80.00 \%)$ & $1(20.00 \%)$ & 0 \\
\hline Increased LDL with hypercholesterolemia & 7 & $5(71.42 \%)$ & 0 & $2(28.57 \%)$ & 0 \\
\hline Low HDL with hypercholesterolemia & 3 & $2(66.66 \%)$ & 0 & $1(33.33 \%)$ & 0 \\
\hline
\end{tabular}

All patients with dyslipidemia, i.e 36 patients were found to have increased TC, of these 5 had increased TG, 7 had increased LDL, 3 had low HDL. In these 36 patients with dyslipidemia $19(52.77 \%)$ were euthyroid, 14(38.88\%) were subclinically hypothyroid, 3(8.33\%) were clinically hypothyroid. Dyslipidemia was not found in thyrotoxic patients. Predominant pattern of dyslipidemia seen in patients with thyroid dysfunction was hypercholesterolemia.

\section{Discussion}

In our study, $36 \%$ of patients had dyslipidemia. $38.9 \%$ of patients with subclinical hypothyroidism $\& 8.3 \%$ of clinical hypothyroid patients were found to have dyslipidemia. Presence of dyslipidemia was statistically associated with thyroid dysfunction with $\mathrm{p}=0.014$. Predominant pattern of dyslipidemia seen was hypercholesterolemia. EPIC Norfolk prospective study found significantly increased concentration of serum total cholesterol (TC), LDL cholesterol (LDLc) and triglycerides in SH women. Similarly in a large cross sectional study an increase of 1.0 $\mathrm{mIU} / \mathrm{L}$ in serum TSH was associated with an average rise in TC values of $0.09 \mathrm{mmol} / \mathrm{L}$ in women. In the latter study the impact of TSH elevation was substantially influenced by age, thus the effect of SH on the serum lipid profile appears more pronounced in women and is also worse with increasing age. Meier et al. ${ }^{[7]}$ reported a reduction of Serum TC by $0.29 \mathrm{mmol} / \mathrm{L}$ of LDLc by $0.33 \mathrm{mmol} / \mathrm{L}$ after 12 months of L-thyroxine replacement. However the effect was most pronounced in patients with baseline serum TSH values $>12 \mathrm{mIU} / \mathrm{L}$. similarly Caracio et al. ${ }^{[8]}$ reported mean 
reductions in serum TC \& LDLc concentrations of $0.47 \&$ $0.41 \mathrm{mmol} / \mathrm{L}$, respectively in a strictly selected group of patients with Hashimoto's thyroiditis \& slightly elevated serum TSH level $(<10 \mathrm{mIU} / \mathrm{L})$. In a subsequent randomised controlled study from the same group L-thyroxine replacement induced a significant improvement of both the lipoprotein profile and the carotid artery intima-media thickness: a widely recognised surrogate index of early atherosclerosis \& CV events. A large meta-analysis in which individual data on more than 50,000 participants from 11 prospective cohorts were collected, demonstrated that CHD mortality was increased in participants with serum TSH $>7 \mathrm{mIU} / \mathrm{L} \&$ that the risk of CHD events was significantly increased once serum TSH $>10 \mathrm{mIU} / \mathrm{L}^{[9]}$. A recent observations analysis of $4500 \mathrm{SH}$ patients from United Kingdom General Practitioner Research Database demonstrated that patients $<70 y$ rs who were started on Lthyroxine had lower CHD events over 8 years of follow up ${ }^{[10]}$. This suggests that L-thyroxine treatment of $\mathrm{SCH}$ is safe and the results are consistent with a modest prognostic benefit from such therapy.

\section{Conclusion}

In our study, dyslipidemia was significantly associated with thyroid dysfunction with predominant pattern being hypercholesterolemia.

\section{References}

1. Schindler AE. Thyroid function and postmenopause, Gynecol Endocrinol 2003;17(1):79-85.

2. Rizos CV et al., Effects of Thyroid Dysfunction on Lipid Profile. The open Cardiovascular Medicine journal Volume 5, 2011.

3. Dr. Pradeep Sharma et al., Hypothyroidism causing dyslipidemia in both subclinical and overt hypothyroidism. Indian Journal of Basic and applied Medical Research Issue-7 Volume- 2.

4. Sulabh Avinash Joshi et al., Screening of Peri and Postmenopausal women for Hypothyroidism Journal of South Asian Federation of Obstetrics \& Gynaecology, Jaypee, Maharashtra 2011;3(1):14-16.

5. Jandee Lee, Woong Youn Chung. Subclinical hypothyroidism, Natural history. Long term clinical effects and treatment, current Topics in Hypothyroidism with Focus on Development, In Tech Journals 2013.

6. Larry Jameson J, Anthony Weetman P. Disorders of Thyroid Gland, Harrison's Principles of Internal Medicine, New Delhi, Mc Graw Hill 2002.

7. Meier C, Staub JJ, Roth CB et al. TSH-controlled L thyroxine therapy reduces cholesterol levels and clinical symptoms in subclinical hypothyroidism: A double blind, placebocontrolled trial (Basel Thyroid Study). J Clin Endocrinol Metab 2001;86:4860-4866.

8. Caraccio N, Ferrannini E, Monzani F. Lipoprotein profile in subclinical hypothyroidism: response to levothyroxine replacement, a randomized placebocontrolled study. J Clin Endocrinol Metab 2002;87:1533-1538.

9. Monzani F, Caraccio N, Kozakowa M et al. Effect of levothyroxine replacement on lipid profile and intimamedia thickness in subclinical hypothyroidism: A double-blind, placebo-controlled study. J Clin Endocrinol.
10. Rodondi N, den Elzen WP, Bauer DC et al. Thyroid Studies Collaboration: Subclinical hypothyroidism and the risk of coronary heart disease and mortality. JAMA 2010;304:1365-1374. 